\title{
Regulating and Helix Path Tracking for Unmanned Aerial Vehicle (UAV) Using Fuzzy Logic Controllers
}

\author{
Mehdi Zare $^{1}$, Jafar Sadeghi ${ }^{2 *}$, Said Farahat ${ }^{3}$, Ehsan Zakeri ${ }^{1}$ \\ ${ }^{1}$ Graduate student, Department of Mechanical Engineering, University of Sistan and \\ Baluchestan, Zahedan, Iran. \\ ${ }^{2}$ Assistant Professor, Department of Chemical Engineering, University of Sistan and \\ Baluchestan, Zahedan, Iran. \\ ${ }^{3}$ Associate Professor, Department of Mechanical Engineering, University of Sistan \\ and Baluchestan, Zahedan, Iran. \\ *sadeghi@eng.usb.ac.ir
}

Article history:

Received July 2014

Accepted September 2014

Available online September 2014

\begin{abstract}
In recent years, most of researchers attempt to replace manpower with robots because of their ability to do repetitive work and also their accuracy in critical condition. Unmanned Aerial Vehicles (UAVs) are attained more attention for their advantages rather than other kind of manned aerial vehicles (MAVs). Quadrotor is a special kind of UAVs with simple mechanical structure and high maneuverability for excellence. Also, quadrotor is a 6-DOF (six degree of freedom) system with high nonlinearity in terms of dynamic equations equipped by four rotors. Consequently, there is the nonlinear under-actuated system with 6-DOF and four angular speeds as system input. Although the high nonlinearity nature of system dynamic cause some difficultly in controlling process, this can be helpful in some cases which quick response is needed. Therefore, the aim of this study is to plan such controller which is able to provide all maneuvers in all reachable direction (maneuvers in all altitude and attitude). In this study, the model of system is considered as Multiple Input-Multiple Output (MIMO) and three fuzzy logic controllers (FLC) are proposed to make system regulated with constant value and tracked with helix path. Finally, results indicate good performance in both regulation and tracking purpose.
\end{abstract}

Keywords: Fuzzy Logic Controller, Nonlinear systems, quadrotor, UAV, under-actuated systems.

\section{Introduction}

In recent modern days, UAVs, quadrotor specially, create some interesting application in control theory. Quadrotor is the most common UAVs which are used in various fields such as engineering, surveying, geography, geology, military application and natural disaster, for instance, inspecting 
pipelines and bridges, photographing remote areas, detecting enemy position and helping natural disaster victims.

Quadrotor is equipped with four rotors, rotating in opposite directions to each other. The rotor's angular velocities are considered as system inputs. Consequently, quadrotor is an under-actuated system by considering all 6-DOF. Moreover, the high nonlinearity nature of system causes some difficulty in controlling system condition.

In last years, researchers have proposed various controllers to make stable flight maneuvers quadrotor model. Two model-based control techniques are applied to quadrotor by S. Bouabdallah et al. [1]. One is a classical approach PID assuming simplified dynamics and another one is a newer technique LQ (linear quadratic), based on more complete model. X-4 flyer is developed with an onboard embedded attitude controller to stabilize flight by Pounds, et al., 2006. Also, a linear SISO (single input-single output) controller was designed to regulate flyer attitude [2]. In 2008s, a nonlinear model of an underactuated quadrotor aerial robot was derived, based on Newton-Euler formalism, and back-stepping based PID control strategy was implemented for the derived model by Ahmad Main, et al., 2008 [3]. A robust control method for a quadrotor mechanisms are developed by Sangyam, et al., 2010. Also, a conventional proportional-integral-derivative (PID) controller and self-tuning PID based on fuzzy logic are compared and analyzed [4]. A fuzzy control is designed and implemented to control a simulation model of the quadrotor with the desired values of the height, roll, pitch and yaw are considered as the inputs, is presented by santo, et al., 2010 [5]. A design of a controller based on the block control technique combined with the super twisting control algorithm for trajectory tracking of a quadrotor helicopter is presented by Luque-Vega, et al., 2012 [6]. A nested loops control architecture in implemented by Nagaty, et al. 2013. A nonlinear Backstepping controller is implemented for the inner stabilization loop and the reference trajectories for the inner loop controller to reach the desired waypoint is generated by the outer loop controller. Moreover, to ensure boundedness of the reference trajectories, a PD controller with a saturation function is used for the outer loop [7]. In [8] an algorithm based on robust fuzzy logic controller to ensure stabilization of the quadrotor is proposed. Also, other control methods are performed to control the quadrotor parameters, For instance, Lyapunov theory [9-12], linear quadratic regulator (LQR) [13], dynamic feedback [14, 15], neural networks [16], reinforcement learning [17], and visual feedback [18-20].

In this study, the nonlinear dynamic model for quadrotor with 6-DOF is derived using Newton-Euler method. Then, three fuzzy logic controllers are implemented to regulation and helix path tracking purpose. In section 2 basic maneuvers of the quadrotor is defined and govern equations is derived using Newton-Euler method. Section 3 is included steady-state equation and control method definition. In this section fuzzy logic controllers is presented. In section 4 performances of the controllers is pictured for regulation and helix path tracking purpose.

\section{Dynamic modeling}

In this section basic maneuvers of the quadrotor is defined, then, quadrotor dynamic modeling is derived using Newton-Euler method and is transformed to steady-state form [25].

First, the possible maneuvers are investigated. These maneuvers are used to planning fuzzy rules in the future. The quad rotor is constructed in two structure, cross structure and plus structure. In this study the plus structure is considered. All maneuvers are shown in figure 1. 


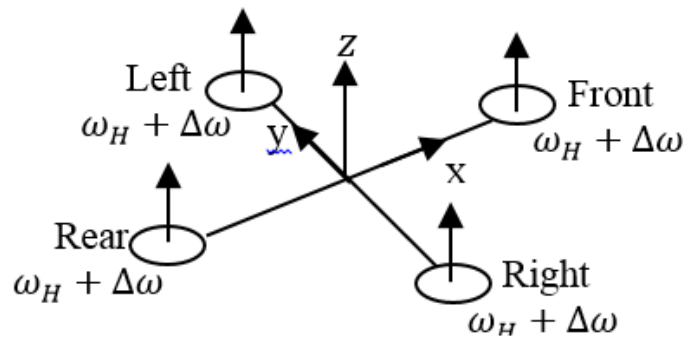

(a)

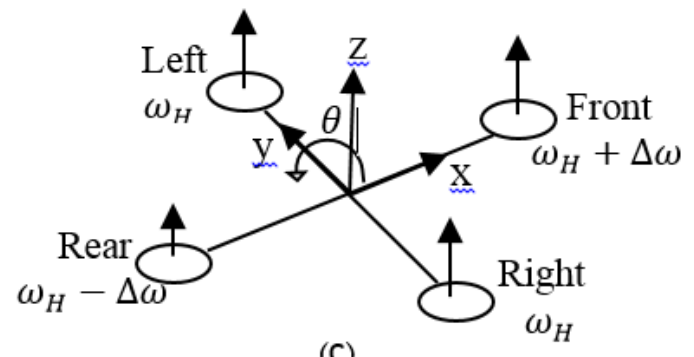

(c)

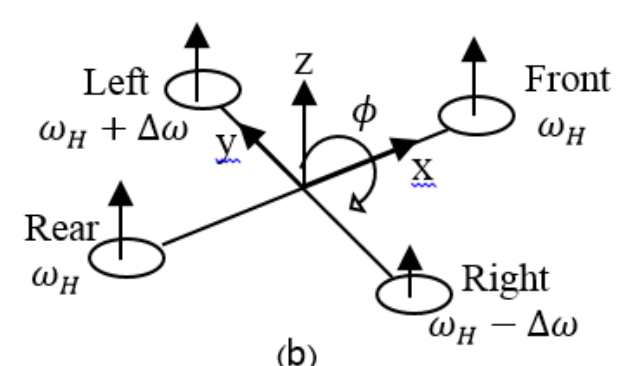

(b)

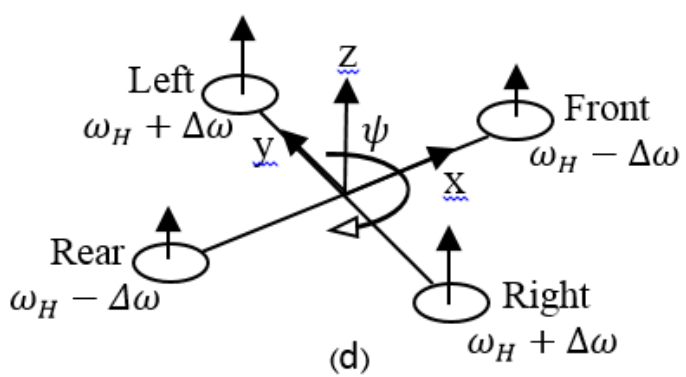

Figure 1. The quadrotor schematic and possible movement

The first maneuver is hovering mode. In this case, all propellers is rotated at the same angular velocity to overcome gravity acceleration and the quadrotor is stabled in the air. The quadrotor is moved up (move down) by increasing (decreasing) the angular velocity of four rotor equally (figure 1.a).

The second maneuver is roll movement which is achieved by moving the rotation around $\mathrm{x}$-axis, namely, by increasing (decreasing) the right while decreasing (increasing) the left propeller's speed. The quadrotor is leaded to linear movement in $\mathrm{x}$-direction by means of pitch maneuver (figure 1.b).

The third maneuver is pitch movement which is achieved by moving the rotation around y-axis, namely, by increasing (decreasing) the rear while decreasing (increasing) the front propeller's speed. The quadrotor is leaded to linear movement in $\mathrm{x}$-direction by means of pitch maneuver (figure 1.c).

The last movement is yaw movement which is achieved by moving the rotation around z-axis, namely, increasing (decreasing) both front while rear and decreasing (increasing) both left and right propeller's speed (figure 1.d).

Two coordinate frames are considered as a reference frames to obtain the dynamic govern equations. One is inertial frame attached to ground (E-frame), and another one is attached to center of mass of the body (B-frame) (figure 2) [26]. These frames are essential for identifying the attitude and altitude of the quadrotor in 6-DOF. For instance, to calculate equations of motion, forces and moments acting on quadrotor, a coordinate frame attached the quadrotor is required. However, the position and speed of quadrotor are evaluated with respect to inertial frame located to the ground.

Also some assumptions are considered for evaluating governs equations, as:

I. The inertia matrix is time-invariant.

II. Control forces are mostly given in B-frame.

III. Advantages of body symmetry can be taken to simplify the equations. 


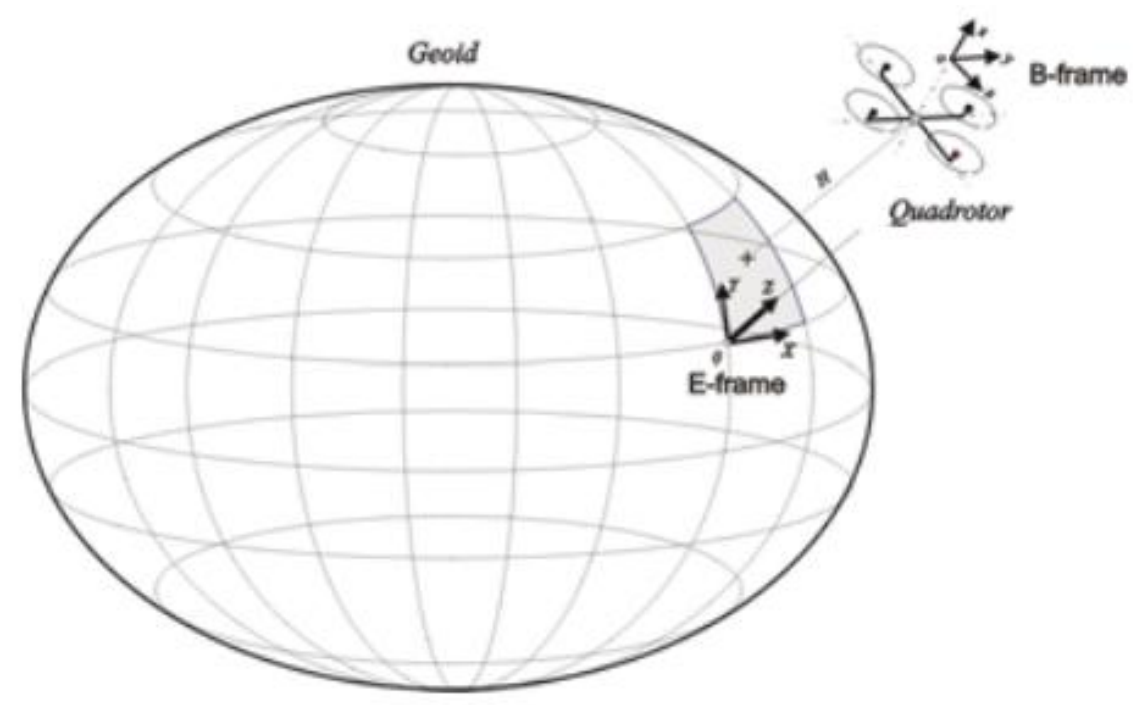

Figure 2. The inertia Frame (E) and the body frame (B).

\subsection{Kinematics of the quadrotor}

A generic 6-DOF rigid body is described as:

$$
\dot{\xi}=J_{\theta} v
$$

Where $\dot{\xi}$ is the generalized velocity vector with respect to E-frame, $v$ is the generalized velocity vector with respect of B-frame and $J_{\theta}$ is the generalized matrix.

$\dot{\xi}$ is composed of the quadrotor linear $\Gamma^{E}(\mathrm{~m})$ and angular $\Theta^{E}(\mathrm{rad})$ position vector with respect to E-frame as:

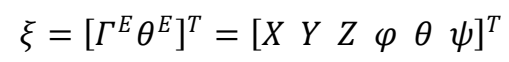

(Error! Bookmark not defined.)

Similarly, $v$ is composed of the quadrotor linear $\mathrm{V}^{\mathrm{B}}\left(\frac{m}{s}\right)$ and angular $\omega^{\mathrm{B}}\left(\frac{\mathrm{rad}}{\mathrm{s}}\right)$ position vector with respect to B-frame as:

$$
J_{\theta}=\left[\begin{array}{cc}
R_{\Theta} & 0_{3 \times 3} \\
0_{3 \times 3} & T_{\Theta}
\end{array}\right]
$$

(Error! Bookmark not defined.)

The rotation $R_{\Theta}$ and the transfer $T_{\Theta}$ matrices are defined as:

$$
\begin{aligned}
& R_{\Theta}=\left[\begin{array}{cc}
c_{\psi} c_{\theta}-s_{\psi} c_{\varphi}+c_{\psi} s_{\theta} s_{\varphi} s_{\psi} s_{\varphi}+c_{\psi} s_{\theta} c_{\varphi} \\
s_{\psi} c_{\theta} c_{\psi} c_{\varphi}+s_{\psi} s_{\theta} s_{\varphi}-c_{\psi} s_{\varphi}+s_{\psi} s_{\theta} c_{\varphi} \\
-s_{\theta} c_{\theta} s_{\varphi} c_{\theta} c_{\varphi}
\end{array}\right] \\
& T_{\Theta}=\left[\begin{array}{ccc}
1 & s_{\varphi} t_{\theta} c_{\varphi} t_{\theta} \\
0 & c_{\varphi} & -s_{\varphi} \\
0 & s_{\varphi} / c_{\theta} c_{\varphi} / c_{\theta}
\end{array}\right]
\end{aligned}
$$

(Error! Bookmark not defined.) 
Where $C_{k}=\cos k, S_{k}=\sin k, t_{k}=\tan k$.

The dynamic of a generic 6-DOF rigid body is obtained by equation (6):

$$
\left[\begin{array}{cc}
m I_{3 \times 3} & 0_{3 \times 3} \\
0_{3 \times 3} & I
\end{array}\right]\left[\begin{array}{c}
\dot{V}^{B} \\
\dot{w}^{B}
\end{array}\right]+\left[\begin{array}{c}
w^{B} \times\left(m V^{B}\right) \\
w^{B} \times\left(I w^{B}\right)
\end{array}\right]=\left[\begin{array}{c}
F^{B} \\
\tau^{B}
\end{array}\right]
$$

(Error! Bookmark not

defined.)

Where the notation $I_{3 \times 3}$ means a 3 times 3 identity matrix. $\dot{V}^{B}\left(\frac{m}{s^{2}}\right)$ is the quadrotor linear acceleration vector, $\dot{\omega}^{b}\left(\frac{\mathrm{rad}}{\mathrm{s}^{2}}\right)$ is the quadrotor angular acceleration vector, $F^{B}(N)$ is the quadrotor forces vector and $\tau^{B}(N . m)$ is the quadrotor torques with respect to B-frame.

Two assumptions are considered in this approach. The first one states that the origin of the body fixed frame, $O_{B}$, is coincident with the center of mass $(\mathrm{COM})$ of the body. Otherwise, another point (COM) should have been taken into account and it would have considerably complicated the body equations. The second one specifies that the axes of the B-frame coincide with the body principal axes of inertia. In this case the inertia matrix I is diagonal and, once again, the body equations become easier.

A generalized force vector $\Lambda$ can be defined as:

$$
\Lambda=\left[F^{B} \tau^{B}\right]=\left[F_{x} F_{y} F_{z} \tau_{x} \tau_{y} \tau_{z}\right]^{T}
$$

Therefore it is possible to rewrite equation (6) in matrix form, as:

$$
M_{B} \dot{v}+C_{B}(v) v=\Lambda
$$

Where $M_{B}$ and $C_{B}$ are system inertia and Coriolis-centripetal matrix, both with respect to B-frame, respectively, is defined as:

$$
\begin{gathered}
\mathrm{M}_{\mathrm{B}}=\left[\begin{array}{cc}
\mathrm{mI}_{3 \times 3} & 0_{3 \times 3} \\
0_{3 \times 3} & \mathrm{I}
\end{array}\right]=\left[\begin{array}{cccccc}
\mathrm{m} & 0 & 0 & 0 & 0 & 0 \\
0 & \mathrm{~m} & 0 & 0 & 0 & 0 \\
0 & 0 & \mathrm{~m} & 0 & 0 & 0 \\
0 & 0 & 0 & \mathrm{I}_{\mathrm{xx}} & 0 & 0 \\
0 & 0 & 0 & 0 & \mathrm{I}_{\mathrm{yy}} & 0 \\
0 & 0 & 0 & 0 & 0 & \mathrm{I}_{\mathrm{zz}}
\end{array}\right] \\
\mathrm{C}_{\mathrm{B}}(v)=\left[\begin{array}{cccccc}
0 & 0 & 0 & 0 & \mathrm{mw} & -\mathrm{mv} \\
0 & 0 & 0 & -\mathrm{mw} & 0 & \mathrm{mu} \\
0 & 0 & 0 & \mathrm{mv} & -\mathrm{mu} & 0 \\
0 & 0 & 0 & 0 & \mathrm{I}_{z z} \mathrm{r} & -\mathrm{I}_{\mathrm{yy}} \mathrm{q} \\
0 & 0 & 0 & -\mathrm{I}_{\mathrm{zz}} \mathrm{r} & 0 & \mathrm{I}_{\mathrm{xx}} \mathrm{p} \\
0 & 0 & 0 & \mathrm{I}_{\mathrm{yy}} \mathrm{q} & -\mathrm{I}_{\mathrm{xx}} \mathrm{p} & 0
\end{array}\right]
\end{gathered}
$$

The overall propeller's speed $\Omega\left(\frac{\mathrm{rad}}{\mathrm{s}}\right)$ and the propeller's speed vector $\Omega\left(\frac{\mathrm{rad}}{\mathrm{s}}\right)$ are defined as:

$$
\Omega=-\Omega_{1}+\Omega_{2}-\Omega_{3}+\Omega_{4} \Omega=\left[\begin{array}{l}
\Omega_{1} \\
\Omega_{2} \\
\Omega_{3} \\
\Omega_{4}
\end{array}\right]
$$


Where $\Omega_{1}\left(\frac{\mathrm{rad}}{\mathrm{s}}\right), \Omega_{2}\left(\frac{\mathrm{rad}}{\mathrm{s}}\right), \Omega_{3}\left(\frac{\mathrm{rad}}{\mathrm{s}}\right)$ and $\Omega_{4}\left(\frac{\mathrm{rad}}{\mathrm{s}}\right)$ are front,right, rear and left propeller's speed, respectively.

The action of the movement vector on the quadrotor dynamics is shown as:

$$
\mathrm{U}_{\mathrm{B}}(\Omega)=\mathrm{E}_{\mathrm{B}} \Omega^{2}=\left[\begin{array}{c}
0 \\
0 \\
\mathrm{U}_{1} \\
\mathrm{U}_{2} \\
\mathrm{U}_{3} \\
\mathrm{U}_{4}
\end{array}\right]=\left[\begin{array}{c}
0 \\
0 \\
\mathrm{~b}\left(\Omega_{1}^{2}+\Omega_{2}^{2}+\Omega_{3}^{2}+\Omega_{4}^{2}\right) \\
\mathrm{bl}\left(\Omega_{4}^{2}-\Omega_{2}^{2}\right) \\
\mathrm{bl}\left(\Omega_{3}^{2}-\Omega_{1}^{2}\right) \\
\mathrm{d}\left(\Omega_{2}^{2}+\Omega_{4}^{2}-\Omega_{1}^{2}-\Omega_{3}^{2}\right)
\end{array}\right]
$$

Where $l(m)$ is the distance between the center of the quadrotor and propellers. $U_{1}, U_{2}, U_{3}$ and $U_{4}$ are the movements vector components introduced in the previous section. The movement matrix $E_{B}$ is shown as:

$$
\mathrm{E}_{\mathrm{B}}=\left[\begin{array}{cccc}
0 & 0 & 0 & 0 \\
0 & 0 & 0 & 0 \\
\mathrm{~b} & \mathrm{~b} & \mathrm{~b} & \mathrm{~b} \\
0 & -\mathrm{bl} & 0 & \mathrm{bl} \\
-\mathrm{bl} & 0 & \mathrm{bl} & 0 \\
-\mathrm{d} & \mathrm{d} & -\mathrm{d} & \mathrm{d}
\end{array}\right]
$$

It is possible to describe the quadrotor dynamics as:

$$
\mathrm{M}_{\mathrm{B}} \dot{v}+\mathrm{C}_{\mathrm{B}}(v) v=\mathrm{G}_{\mathrm{B}}(\xi)+\mathrm{O}_{\mathrm{B}}(v) \Omega+\mathrm{E}_{\mathrm{B}} \Omega^{2}
$$

By rearranging equation (14) it is possible to isolate the derivate of the generalized velocity vector with respect to B-frame $\dot{v}$ as:

$$
\dot{v}=M_{B}^{-1}\left(-C_{B}(v) v+G_{B}(\xi)+O_{B}(v) \Omega+E_{B} \Omega^{2}+D_{B}\left(C_{x, y, z}, v\right) v\right)
$$

Where $G_{B}$ and $O_{B}$ defined as:

$$
\left.\begin{array}{c}
G_{B}=\left[\begin{array}{c}
F_{G}^{B} \\
0_{3 \times 1}
\end{array}\right]=\left[\begin{array}{c}
R_{\theta}^{-1} F_{G}^{E} \\
0_{3 \times 1}
\end{array}\right]=\left[R_{\theta}^{-1}\left[\begin{array}{c}
0 \\
0 \\
-m g
\end{array}\right]=\left[\begin{array}{c}
m g s_{\theta} \\
-m g c_{\theta} s_{\varphi} \\
-m g c_{\theta} c_{\varphi} \\
0 \\
0
\end{array}\right]\right. \\
0
\end{array}\right]=\left[\begin{array}{c}
0_{3 \times 1} \\
0_{3 \times 1}(v) \Omega=-\sum_{k=1}^{4} J_{T P}\left(w^{B} \times\left[\begin{array}{l}
0 \\
0 \\
1
\end{array}\right]\right) \times(-1)^{k} \Omega_{k}=\left[\begin{array}{c}
-\dot{\theta} \\
\dot{\phi} \\
0
\end{array}\right] \Omega\left[\begin{array}{cccc}
0 & 0 & 0 \\
0 & 0 & 0 & 0 \\
0 & 0 & 0 & 0 \\
q & -q & q & -q \\
-p & p & -p & p \\
0 & 0 & 0 & 0
\end{array}\right] \Omega
\end{array}\right.
$$

Equation (18) shows the previous expression not in a matrix form, but in a system of equations. 


$$
\left\{\begin{array}{c}
\dot{\mathrm{u}}=(\mathrm{vr}-\mathrm{wq})+\mathrm{gs}_{\theta} \\
\dot{\mathrm{v}}=(\mathrm{wp}-\mathrm{ur})-\mathrm{gc}_{\theta} \mathrm{s}_{\varphi} \\
\dot{\mathrm{w}}=(\mathrm{uq}-\mathrm{vp})-\mathrm{g} \mathrm{c}_{\theta} \mathrm{c}_{\varphi}+\frac{\mathrm{U}_{1}}{\mathrm{~m}} \\
\dot{\mathrm{p}}=\frac{\mathrm{I}_{\mathrm{yy}}-\mathrm{I}_{\mathrm{zz}}}{\mathrm{I}_{\mathrm{xx}}} \mathrm{qr}-\frac{\mathrm{J}_{\mathrm{TP}}}{\mathrm{I}_{\mathrm{xx}}} \mathrm{q} \Omega+\frac{\mathrm{U}_{2}}{\mathrm{I}_{\mathrm{xx}}} \\
\dot{\mathrm{q}}=\frac{\mathrm{I}_{\mathrm{zz}}-\mathrm{I}_{\mathrm{Xx}}}{\mathrm{I}_{\mathrm{yy}}} \mathrm{pr}+\frac{\mathrm{J}_{\mathrm{TP}}}{\mathrm{I}_{\mathrm{yy}}} \mathrm{P} \Omega+\frac{\mathrm{U}_{3}}{\mathrm{I}_{\mathrm{yy}}} \\
\dot{\mathrm{r}}=\frac{\mathrm{I}_{\mathrm{xx}}-\mathrm{I}_{\mathrm{yy}}}{\mathrm{I}_{\mathrm{zz}}} \mathrm{pq}+\frac{\mathrm{U}_{4}}{\mathrm{I}_{\mathrm{zz}}}
\end{array}\right.
$$

Where the propeller's speeds input are given through the equation (19).

$$
\left\{\begin{array}{c}
\mathrm{U}_{1}=\mathrm{b}\left(\Omega_{1}^{2}+\Omega_{2}^{2}+\Omega_{3}^{2}+\Omega_{4}^{2}\right) \\
\mathrm{U}_{2}=\mathrm{bl}\left(\Omega_{4}^{2}-\Omega_{2}^{2}\right) \\
\mathrm{U}_{3}=\mathrm{bl}\left(\Omega_{3}^{2}-\Omega_{1}^{2}\right) \\
\mathrm{U}_{4}=\mathrm{d}\left(-\Omega_{1}^{2}+\Omega_{2}^{2}-\Omega_{3}^{2}+\Omega_{4}^{2}\right) \\
\Omega=-\Omega_{1}+\Omega_{2}-\Omega_{3}+\Omega_{4}
\end{array}\right.
$$

The equation (19) states the quadrotor dynamics in the body fixed frame. By introducing hybrid $\mathrm{H}$-frame as combination of two body fixed frame (B-frame) and inertia frame (E-frame), it can be useful to express the quadrotor dynamics with respect to a hybrid system composed of linear equations in the E-frame and angular equations in the B-frame.

The quadrotor generalized velocity vector with respect to $\mathrm{H}$-frame defined as:

$$
\xi=\left[\dot{\Gamma}^{\mathrm{E}} \omega^{\mathrm{B}}\right]^{\mathrm{T}}=[\dot{\mathrm{X} Y \dot{Z}} \text { p q r }]^{\mathrm{T}}
$$

The dynamics of the system in the H-frame can be written in a matrix form as:

$$
\mathrm{M}_{\mathrm{H}} \dot{\xi}+\mathrm{C}_{\mathrm{H}}(\xi) \xi=\mathrm{G}_{\mathrm{H}}+\mathrm{O}_{\mathrm{H}}(\xi) \Omega+\mathrm{E}_{\mathrm{H}}(\xi) \Omega^{2}
$$

Where, $\dot{\xi}$ is the quadrotor generalized acceleration vector in the $\mathrm{H}$-frame. $M_{H}$ and $O_{H}$ are the same as in the B-frame. By this definition $C_{H}, G_{H}$ and $E_{H}$ are:

$$
C_{H}(\xi)=\left[\begin{array}{rrrrcc}
0 & 0 & 0 & 0 & 0 & 0 \\
0 & 0 & 0 & 0 & 0 & 0 \\
0 & 0 & 0 & 0 & 0 & 0 \\
0 & 0 & 0 & 0 & I_{z z} r & -I_{y y} q \\
0 & 0 & 0 & -I_{z z} r & 0 & I_{x x} p \\
0 & 0 & 0 & I_{y y} q & -I_{x x} p & 0
\end{array}\right]
$$




$$
\begin{gathered}
G_{H}=\left[\begin{array}{c}
F_{G}^{H} \\
0_{3 \times 1}
\end{array}\right]=\left[\begin{array}{c}
0 \\
0 \\
-m g \\
0 \\
0 \\
0
\end{array}\right] \\
E_{H}(\xi) \Omega^{2}=\left[\begin{array}{cc}
R_{\theta} & 0_{3 \times 3} \\
0_{3 \times 3} & I_{3 \times 3}
\end{array}\right] E_{B} \Omega^{2}=\left[\begin{array}{c}
\left(s_{\psi} s_{\varphi}+c_{\psi} s_{\theta} c_{\varphi}\right) U_{1} \\
\left(-c_{\psi} s_{\varphi}+s_{\psi} s_{\theta} c_{\varphi}\right) U_{1} \\
\left(c_{\theta} c_{\varphi}\right) U_{1} \\
U_{2} \\
U_{3} \\
U_{4}
\end{array}\right]
\end{gathered}
$$

Equation (25) shows equation (18) not in matrix form, but in system equations, as:

$$
\left\{\begin{array}{c}
\ddot{X}=\left(s_{\psi} s_{\varphi}+c_{\psi} s_{\theta} c_{\varphi}\right) \frac{U_{1}}{m} \\
\ddot{Y}=\left(-c_{\psi} s_{\varphi}+s_{\psi} s_{\theta} c_{\varphi}\right) \frac{U_{1}}{m} \\
\ddot{Z}=-g+\left(c_{\theta} c_{\varphi}\right) \frac{U_{1}}{m} \\
\dot{p}=\frac{I_{y y}-I_{z z}}{I_{x x}} q r-\frac{J_{T P}}{I_{x x}} q \Omega+\frac{U_{2}}{I_{x x}} \\
\dot{q}=\frac{I_{z z}-I_{x x}}{I_{y y}} p r+\frac{J_{T P}}{I_{y y}} P \Omega+\frac{U_{3}}{I_{y y}} \\
\dot{r}=\frac{I_{x x-I}-I_{y y}}{I_{z z}} p q+\frac{U_{4}}{I_{z z}}
\end{array}\right.
$$

\section{Controller design}

By introducing $\dot{X}=f(X, U)$, where $X=\left(x_{1}, x_{2}, \ldots, x_{12}\right)^{T}$, the steady-state form of previous equations are obtained as: 


$$
X=\left(\begin{array}{c}
x_{1} \\
x_{2} \\
x_{3} \\
x_{4} \\
x_{5} \\
x_{6} \\
x_{7} \\
x_{8} \\
x_{9} \\
x_{10} \\
x_{11} \\
x_{12}
\end{array}\right)=\left(\begin{array}{c}
x \\
\dot{x} \\
y \\
\dot{y} \\
z \\
\dot{z} \\
\phi \\
\dot{\phi} \\
\theta \\
\dot{\theta} \\
\psi \\
\dot{\psi}
\end{array}\right) \rightarrow f(X, U)=\left(\begin{array}{c}
x_{2} \\
\left(\cos \left(x_{7}\right) \sin \left(x_{9}\right) \cos \left(x_{11}\right)+\sin \left(x_{7}\right) \sin \left(x_{11}\right)\right) \frac{U_{1}}{m} \\
x_{4} \\
\left(\cos \left(x_{7}\right) \sin \left(x_{9}\right) \sin \left(x_{11}\right)-\sin \left(x_{7}\right) \cos \left(x_{11}\right)\right) \frac{U_{1}}{m} \\
x_{6} \\
-g+\left(\cos \left(x_{7}\right) \cos \left(x_{9}\right)\right) \frac{U_{1}}{m} \\
x_{8} \\
x_{12} x_{10}\left(\frac{I_{y}-I_{z}}{I_{x}}\right)-\frac{J_{r}}{I_{x}} x_{10} \Omega+\frac{l}{I_{x}} U_{2} \\
x_{10} \\
x_{12} x_{8}\left(\frac{I_{z}-I_{x}}{I_{y}}\right)-\frac{J_{r}}{I_{y}} x_{8} \Omega+\frac{l}{I_{y}} U_{3} \\
x_{12} \\
x_{10} x_{8}\left(\frac{I_{x}-I_{y}}{I_{z}}\right)+\frac{l}{I_{z}} U_{4}
\end{array}\right)
$$

These equations are used to implementing the quadrotor system in MATLAB@ SIMULINK.

\subsection{Fuzzy logic controllers Description}

Since fuzzy logic is introduced by Zadeh [21], it has been applied to various field of science, such as engineering, psychology, manufacturing, medicine, etc. In engineering, fuzzy logic is utilized to controlling systems [22, 23and 24]. Fuzzy logic controller has a great advantage in compare with some other control methods which heavily depends on the mathematical model of the system, specifically in dealing with nonlinear system with complicated model and various uncertainties. Fuzzy logic controller can be implemented directly in light of the knowledge based on the behavior of the system in hand. This information is often obtained through experience and common sense, regardless of mathematical model. For example, the quadrotor leads to y-direction by slightly roll movement. So, humans try to make a set of rules without paying any attention to the mathematical model of the system. Fuzzy logic controller tries to imitate this human sense and induces system to match the desired values without considering the order of system. Therefore, it is useful to underactuated systems specially.

\subsubsection{Implementing controllers}

The quadrotor is an under-actuated system with four motors as actuators and 6-DOF. There are two main challenges to controlling all system conditions. One is controlling quadrotor's position and the other one is attitude stabilization, i.e., roll, pitch and yaw angles. There are four control variable, namely, $x, y$ and $\mathrm{z}$ positions and yaw angle. The controllers are designed to set these variables converge to their respective desired values, while the roll and pitch angles as close to zero as possible.

Three fuzzy logic controllers are designed to make system imitating desired signals. These three controller are connected to each other such one controller is fed by two other controllers output. The schematic of controllers dealing with the quadrotor is shown below (figure 3 ). 


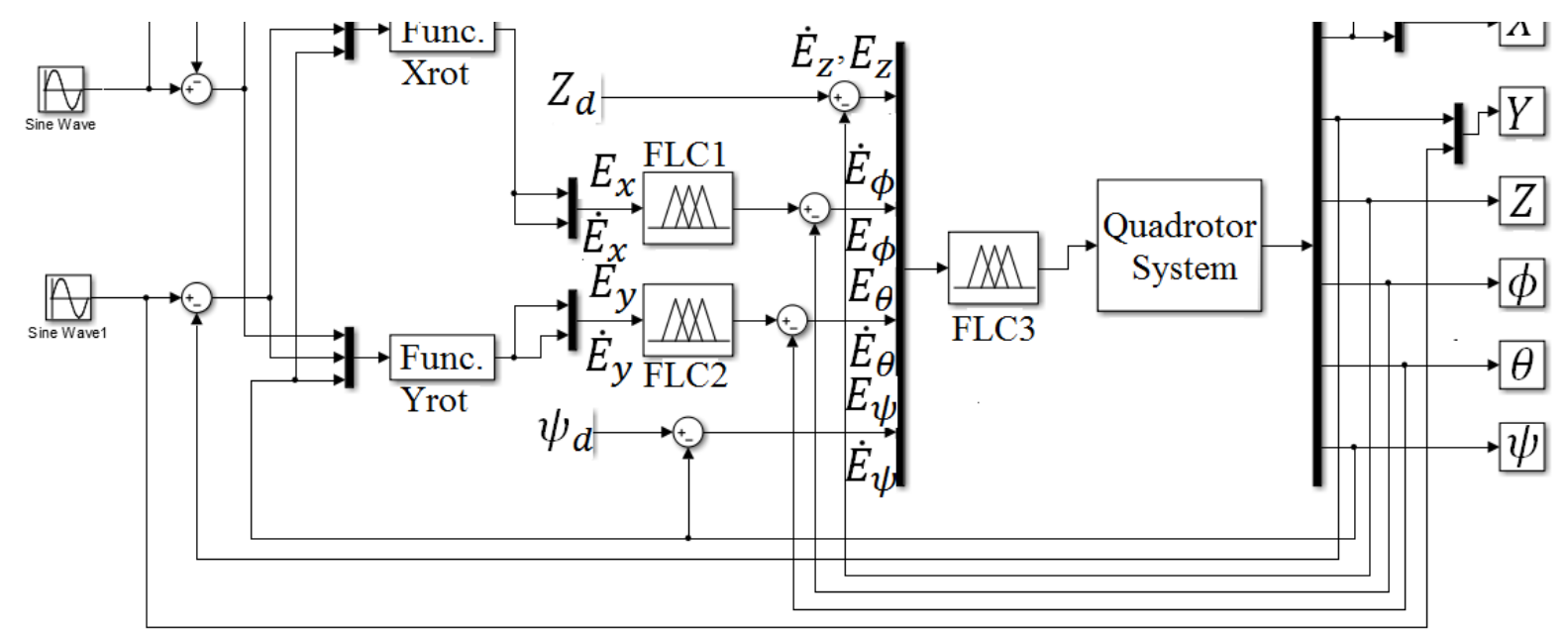

Figure 3. Schematic of controllers dealing with the quadrotor system

In this study, the Tagaki-Sugeno-Kang (TSK) fuzzy model is used to define fuzzy rules. The difference of desired value and system output and its rate are considered as controller inputs in each controller. The procedure is that the first controller is designed to imitate desired sinusoidal wave with respect to $x$ by appropriate change of $\theta$. Same, the second controller is designed to imitate desired sinusoidal wave with respect to y by appropriate change of $\phi$. The error and error rates of these two yielded variable, $\theta, \phi$ and two other variable, $\mathrm{z}, \psi$ variables are used as the third controller input. Then the third controlleradjusts the four rotors speed to the system. As mentioned, the system is under-actuated by four rotors speed and 6DOF. The lift force is applied to the quadrotor via increasing (or decreasing) propeller speeds. The speed in where the quadrotor is stabled in hovering mode is considered as based (zero value) to introduce fuzzy logic rules. Then by dividing error and error rate to five intervals as linguistic variables, medium negative $(\mathrm{MN})$, small negative (SN), zero, small positive (SP), medium positive (MP), the propeller speed is divided into nine intervals as linguistic variables. These nine intervals are incrementally sorted as very big negative (VBN), big negative (BN), medium negative (MN), small negative ( $\mathrm{SN}$ ), zero, small positive (SP), medium positive (MP), big positive (BP), very big positive (VBP). Table 1 is presented to show error and error rate value and yielded propeller speed which is used to design fuzzy logic rules in all three controllers.

Table 1.The errors and error rates and yielded propeller's speeds.

\begin{tabular}{cccccc}
\hline MN & VBN & BN & MN & SN & Zero \\
SN & BN & MN & SN & Zero & SP \\
Zero & MN & SN & Zero & SP & MP \\
SP & SN & Zero & SP & MP & BP \\
MP & Zero & SP & MP & BP & VBP \\
\hline
\end{tabular}


According to table 1, the fuzzy logic rules are obtained as:

$X$-controller: if the error is medium negative and the error rate is medium negative then the $\theta$ is very big negative.

$Y$-controller: if the error is medium negative and the error rate is medium negative then the $\phi$ is very big negative.

Also, there are four branches of rules for the third controller, as (table 2):

Table 2. The rules of third controller

\begin{tabular}{crrrrrr}
\hline parameter & $E$ & $\dot{E}$ & $\omega_{1}$ & $\omega_{2}$ & $\omega_{3}$ & $\omega_{4}$ \\
\hline$Z$ & MN & MN & VBN & VBN & VBN & VBN \\
$\phi$ & MN & MN & No change & VBN & No change & VBN \\
$\theta$ & MN & MN & VBN & No change & VBN & No change \\
$\psi$ & MN & MN & VBN & No change & VBN & No change \\
\hline
\end{tabular}

The membership functions of the error and error rate of the controllers input are considered as figure 3 to figure 8. According to figures the membership functions (MFs) are set as triangular and trapezoidal.
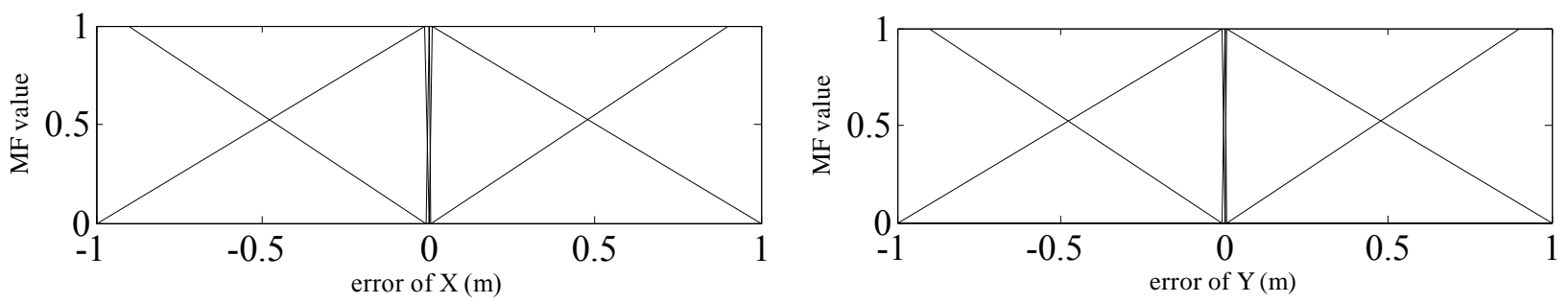

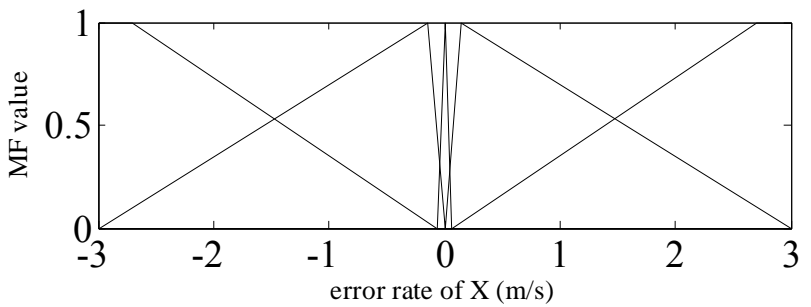

Figure 4. Error and error rate of $X$

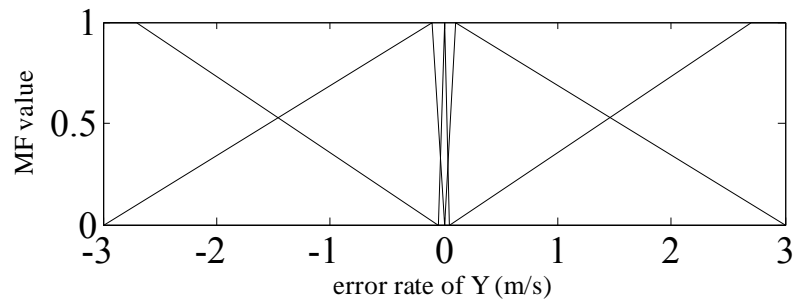

Figure 5. Error and error rate of $Y$ 

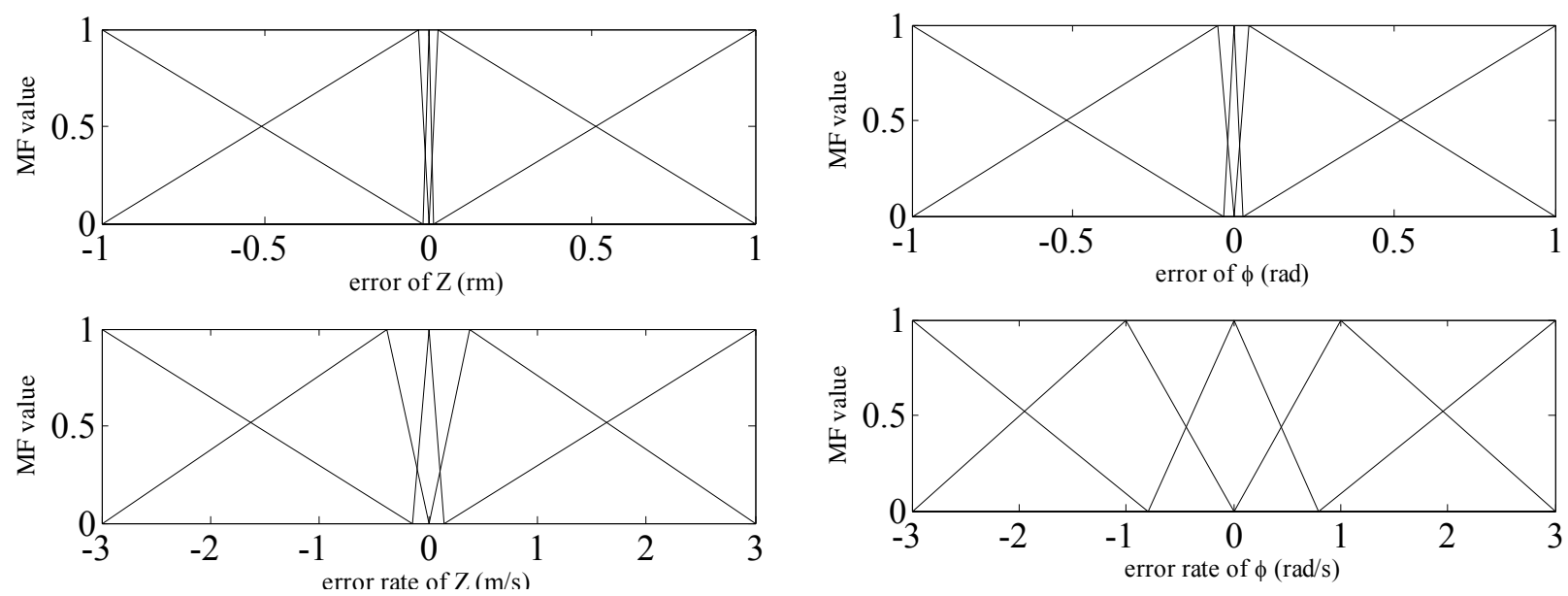

Figure 6. Error and error rate of $Z$

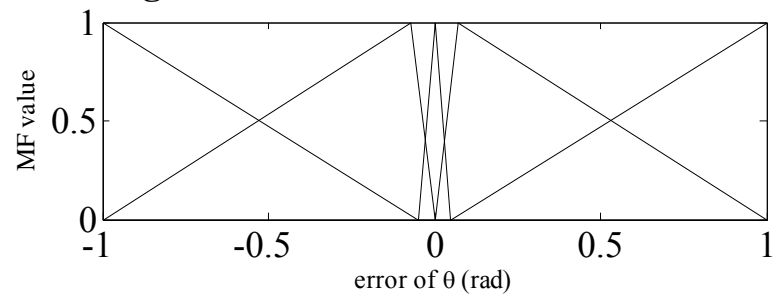

Figure 7. Error and error rate of $\phi$
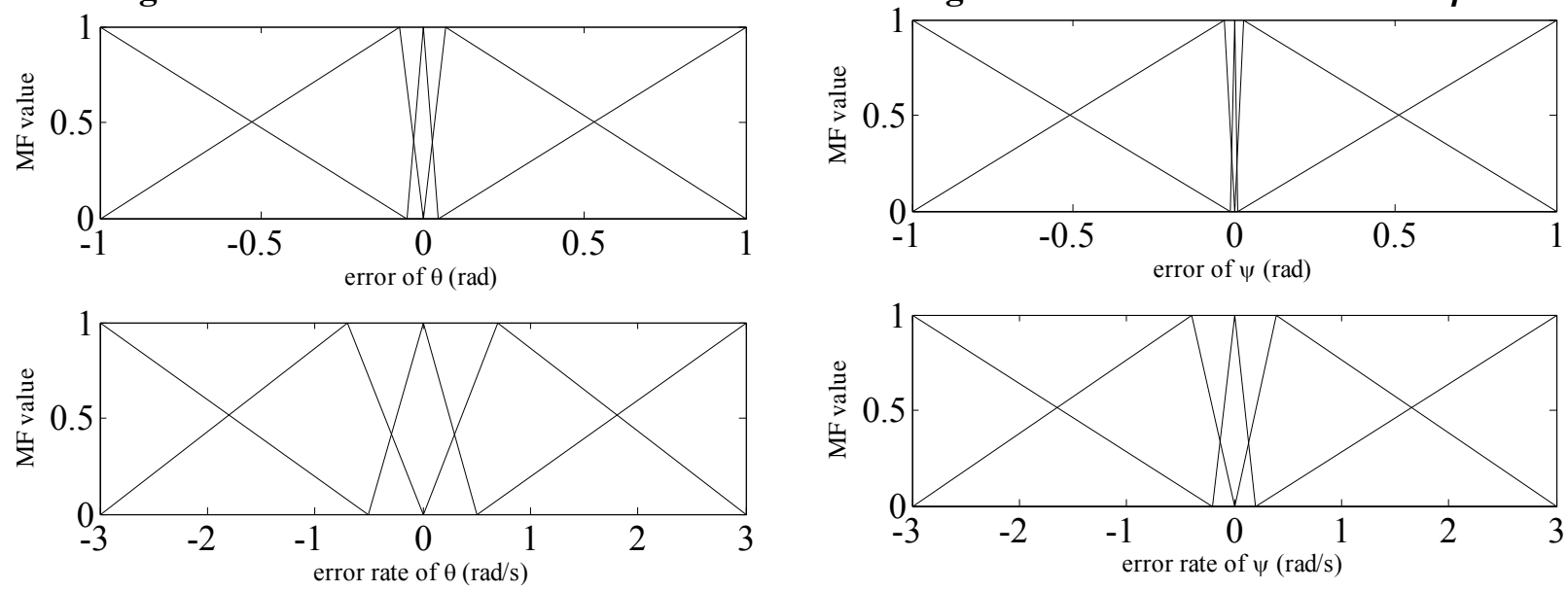

Figure 8. Error and error rate of $\theta$

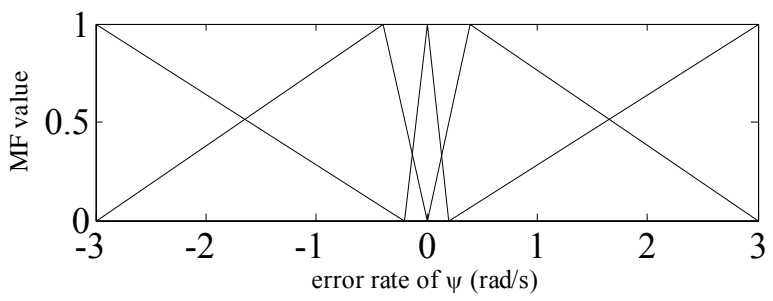

Figure 9. Error and error rate of $\psi$

\section{Simulation results}

In this section, the results of regulating and helix path tracking of the quadrotor are shown. The earlier equations are used to implementing the model of the quadrotor in MATLAB Simulink, as shown below. 


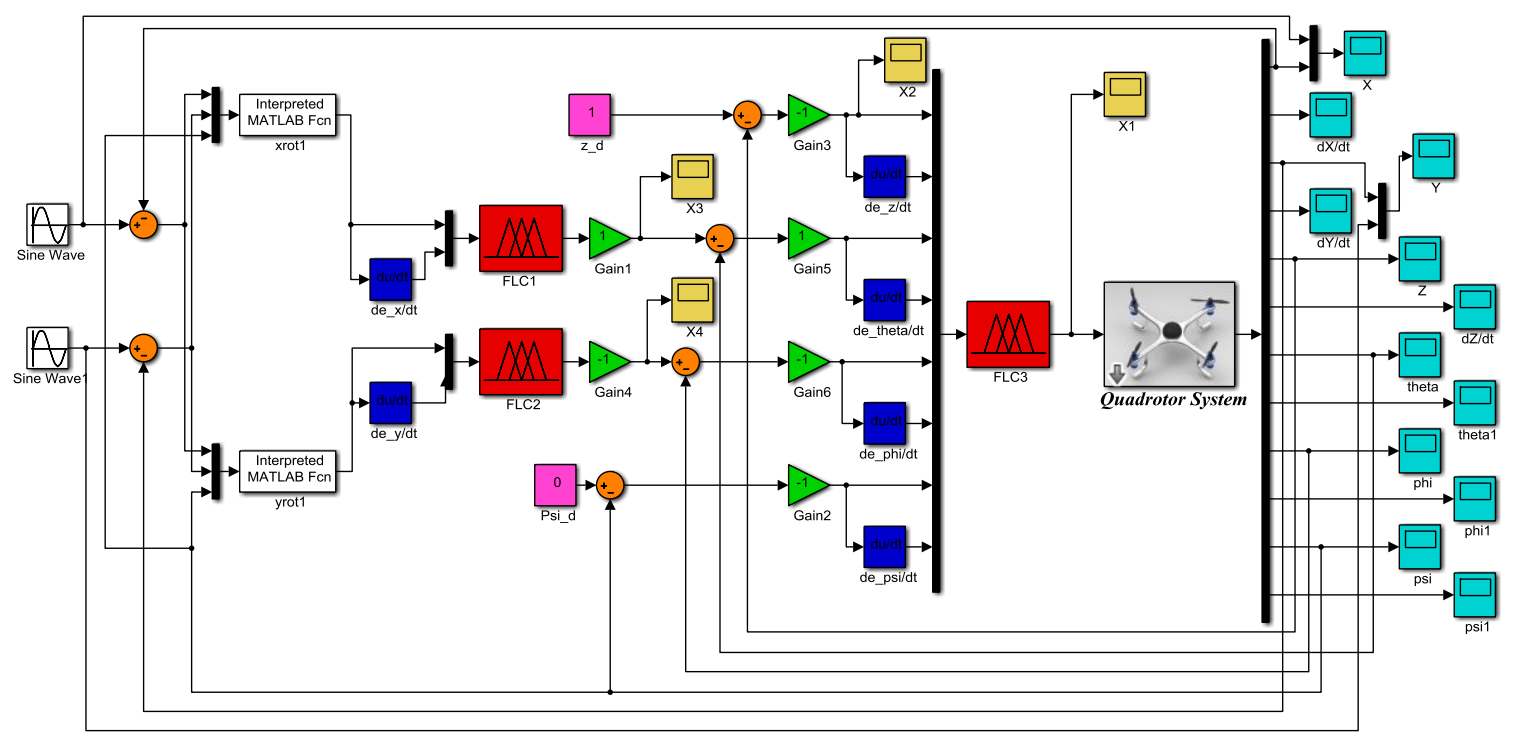

Figure 10. MATLAB Simulink block diagram of the quadrotor model

Figure 11 shows the regulation of the quadrotor states. The desired vector is considered as:

$\left[\begin{array}{llll}X & Y & Z & \psi\end{array}\right]=\left[\begin{array}{llll}0.1 \mathrm{~m} & 0.1 \mathrm{~m} & 0.1 \mathrm{~m} & 0.1 \mathrm{rad}\end{array}\right]$
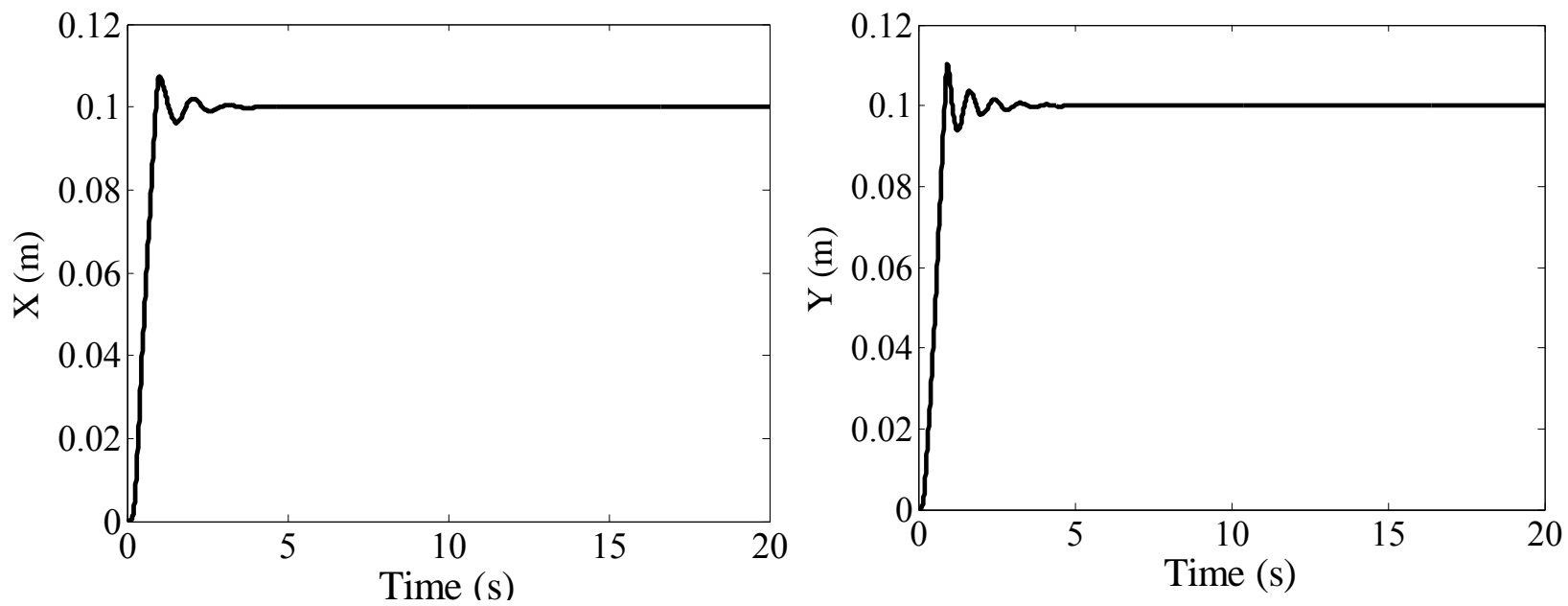

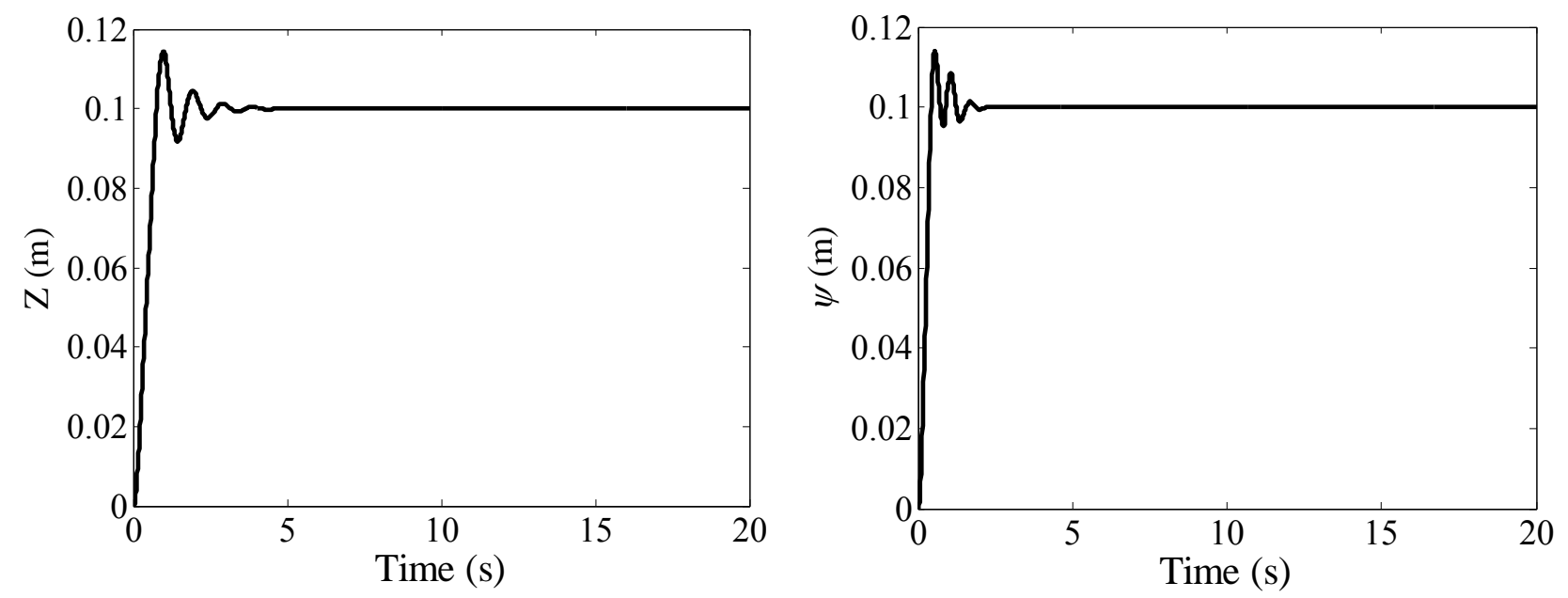

Figure 11. Regulation of quadrotor to the mentioned desired states

Figure 12 is presented to show an appropriate angular speeds which are needed to achieve this position (namely, $\left[\begin{array}{llll}X & Y & Z & \psi\end{array}\right]=\left[\begin{array}{llll}0.1 \mathrm{~m} & 0.1 \mathrm{~m} & 0.1 \mathrm{~m} & 0.1 \mathrm{rad}\end{array}\right]$ ), while these angular speeds are supplied with four motors. There is just first 5 second to show actuators performance clearly.

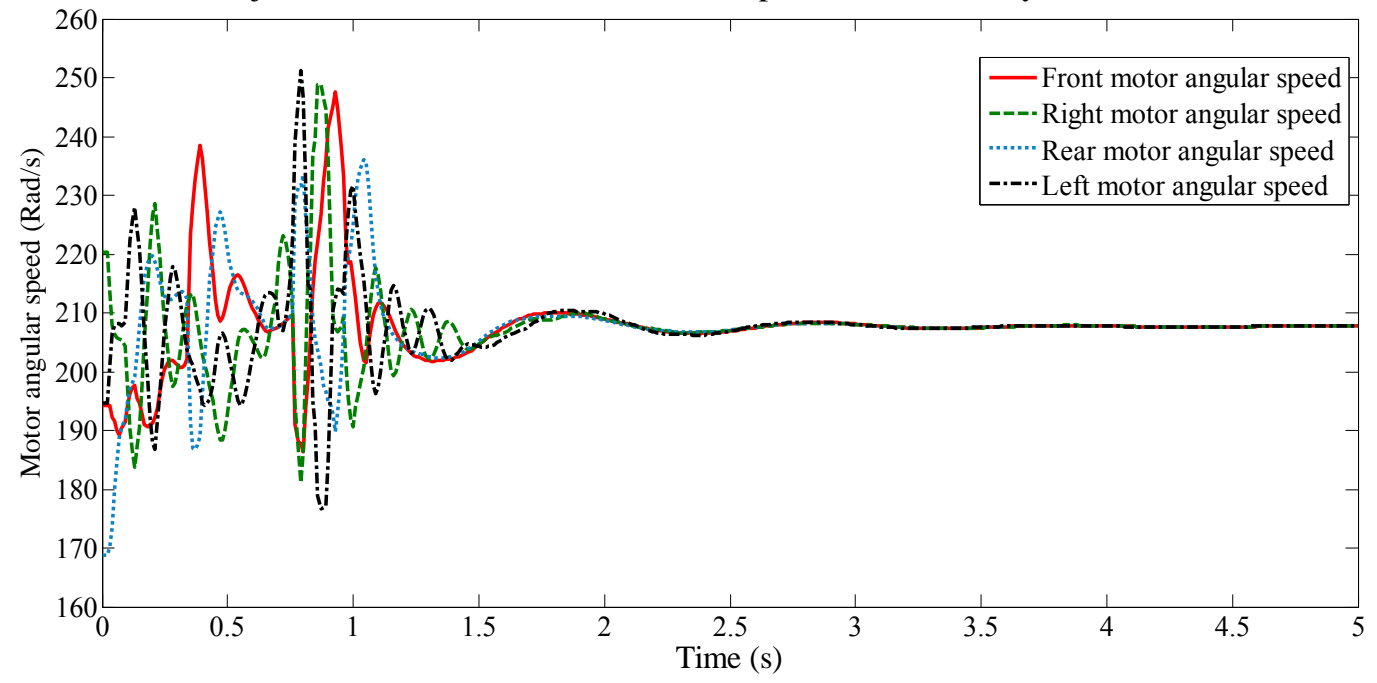

Figure 12. Variations of four motor Angular speedsfor regulate purpose.

Applied roll and pitch variations to imitate the desired mention input are as below:
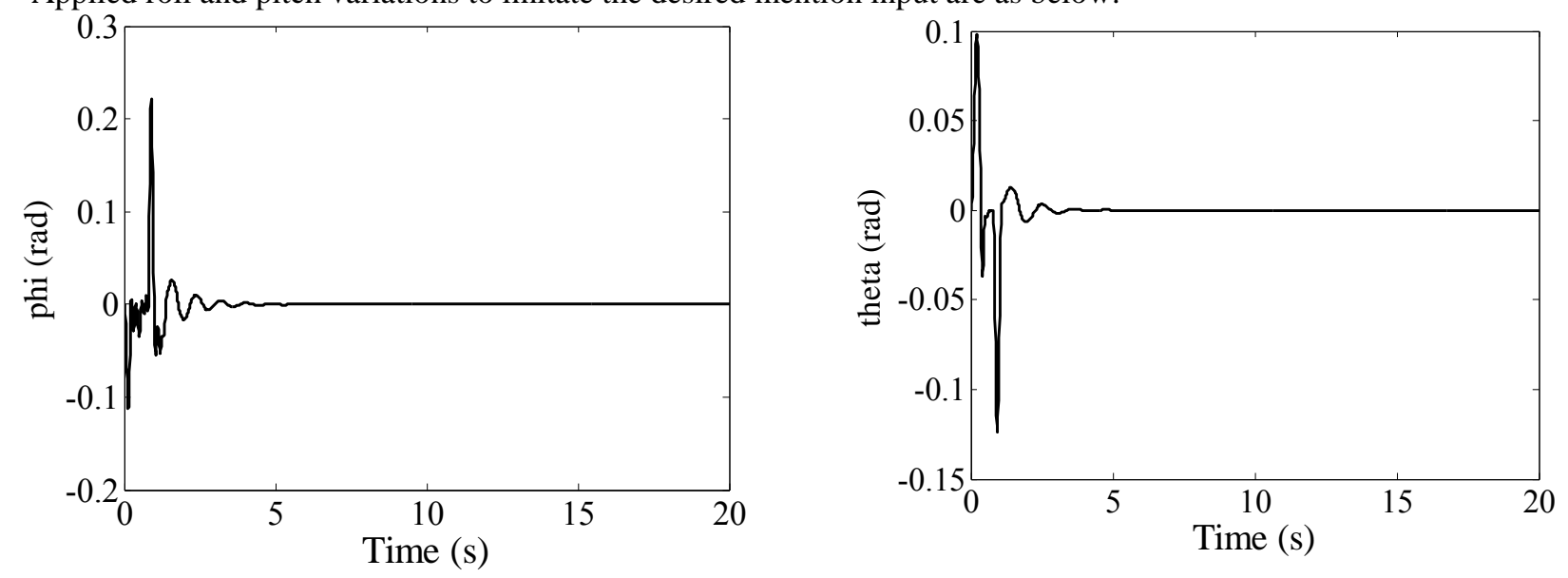


\section{Figure 13. Pitch and roll maneuvers to achieve mentioned position.}

Figures 14 to 17 are presented to show the controllers ability to tracking the desired path in $\mathrm{x}, \mathrm{y}, \mathrm{z}$ and $\psi$, respectively. As mentioned, the desired path is considered as a circle path developing in $\mathrm{Z}$ direction by the ramp input.

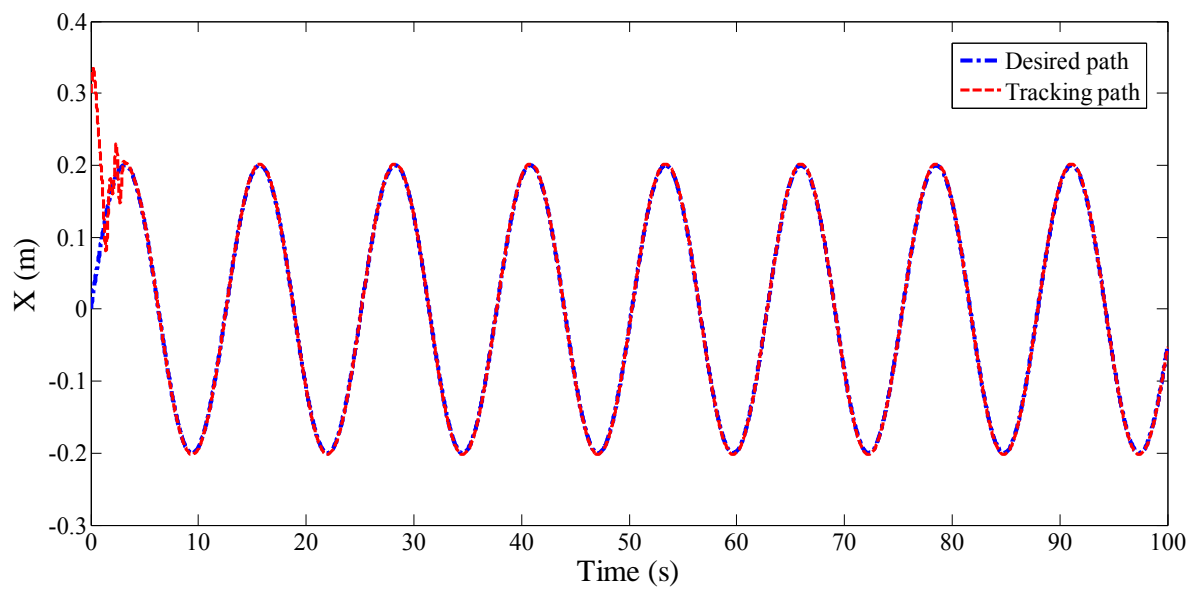

Figure 14. X-tracking of the quadrotor.

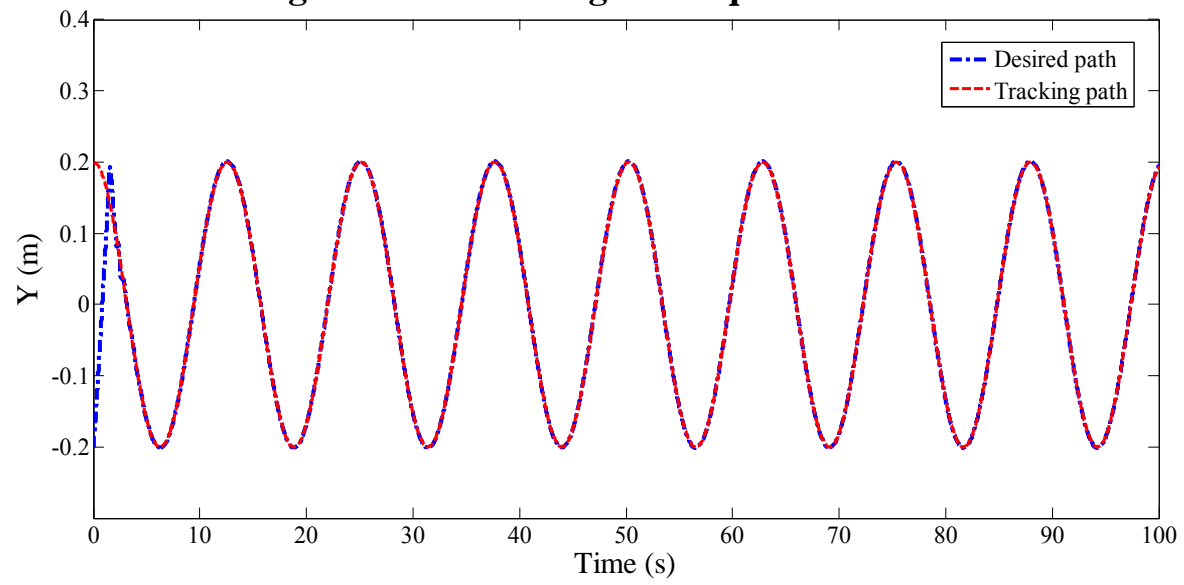

Figure 15. Y-tracking of the quadrotor.

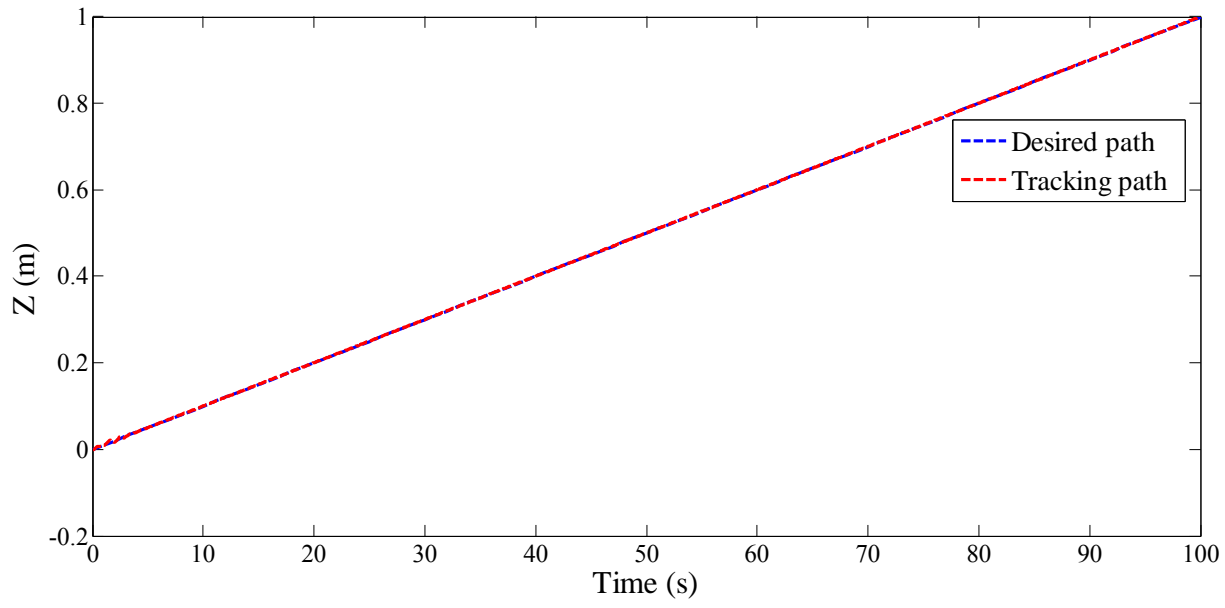

Figure 16. Z-tracking of the quadrotor. 
Figure 17 is presented to show an appropriate angular speeds which are needed to provide path tracking, while these angular speeds is supplied with four motors. There is just first 5 second to show actuators performance clearly.

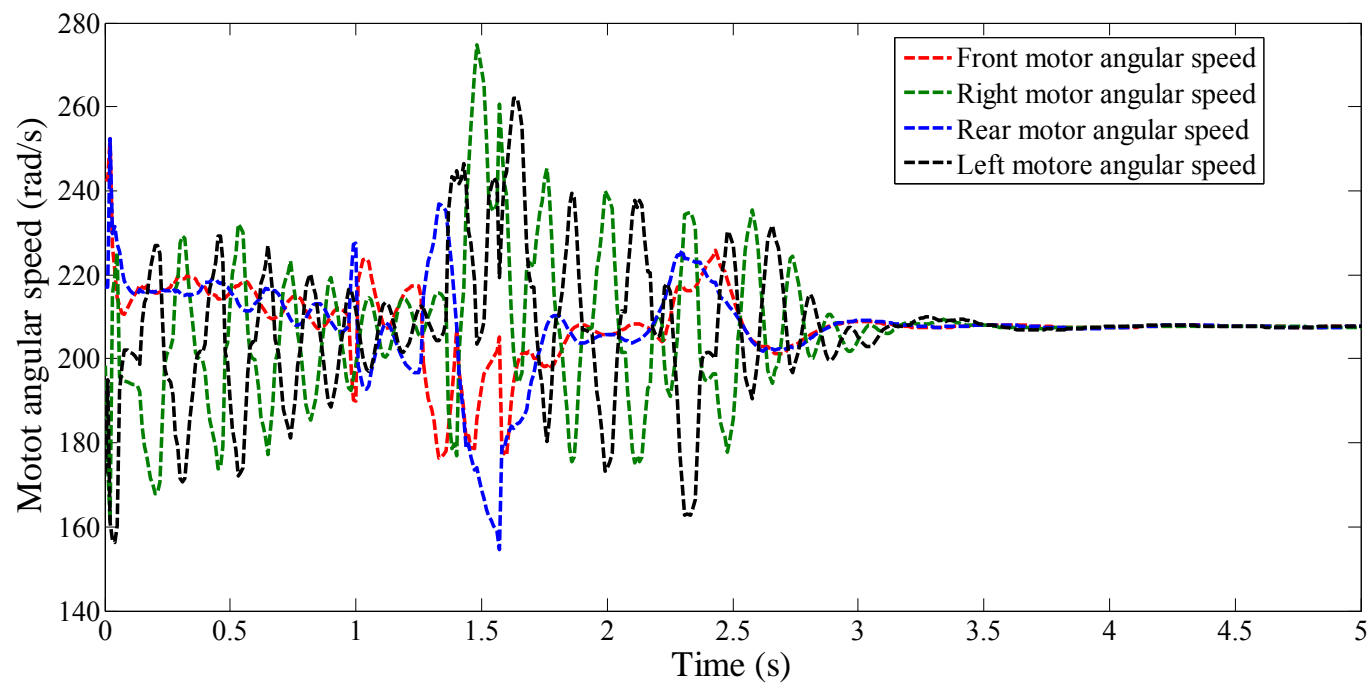

Figure 17. Variations of four motor Angular speeds for tracking purpose.

The role, pitch and yaw angle variations during helix path tracking are presented in figures 18 to 20 .

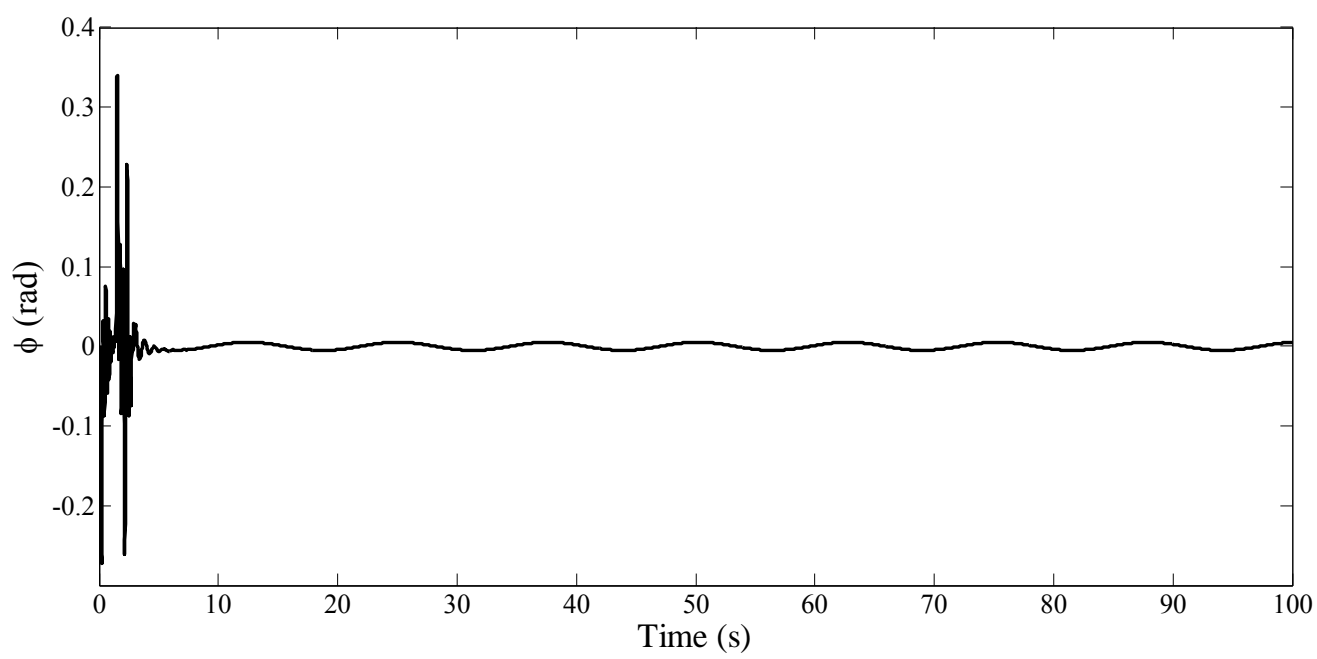

Figure 18. The role angle variations during helix path tracking 


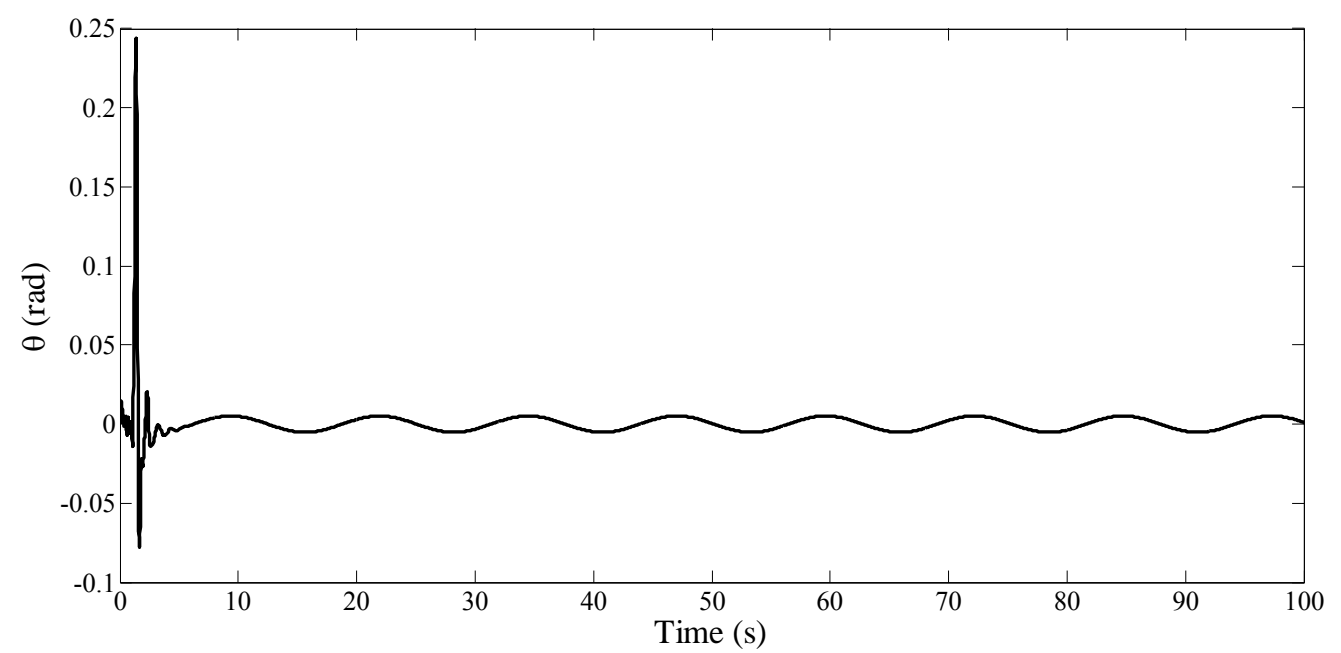

Figure 19. The pitch angle variations during helix path tracking

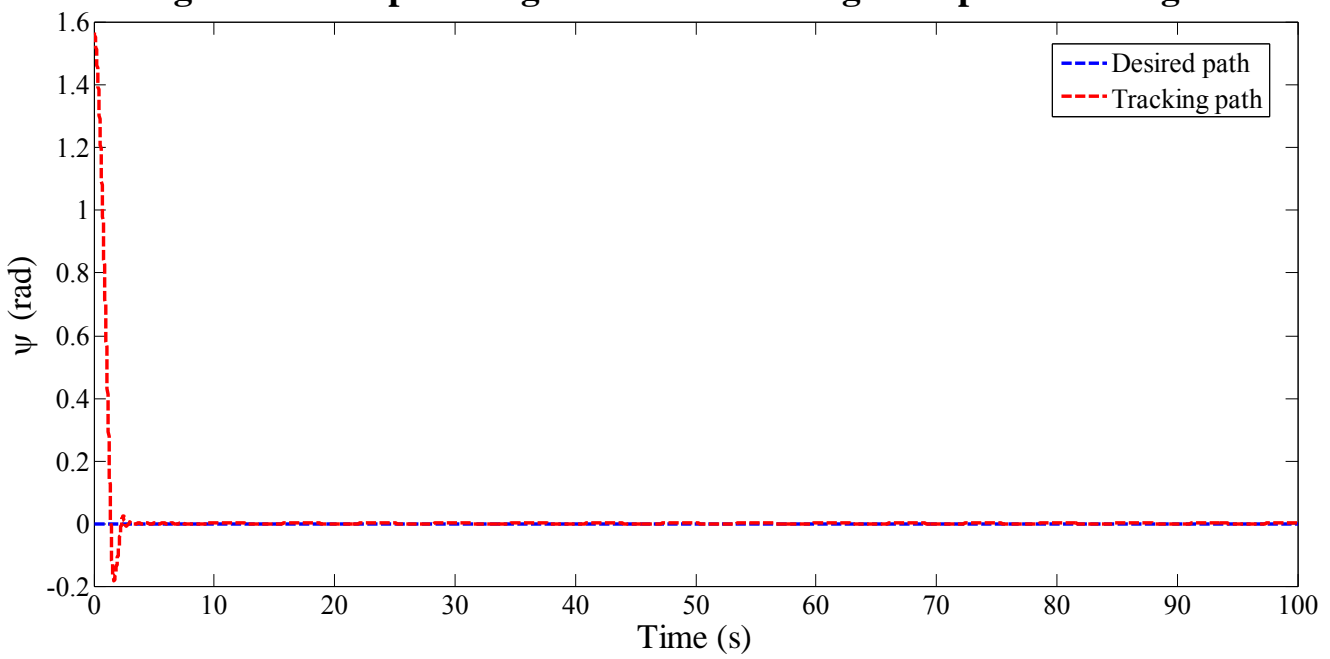

Figure 20. The yaw angle variations during helix path tracking

Finally, the quadrotor is tracked the helical path by considering sinusoidal signals as desired input (figure 21).

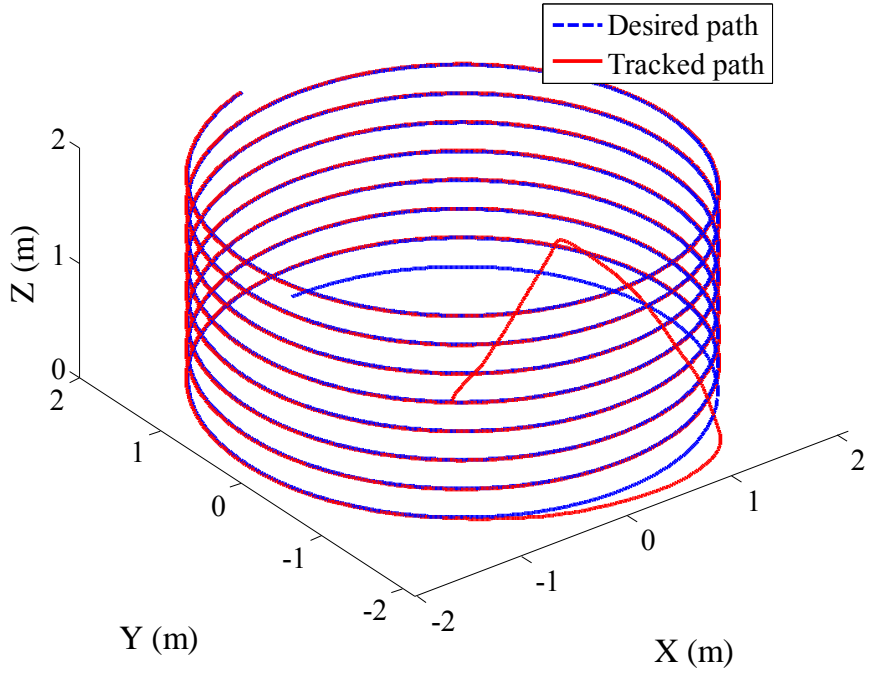

Figure 21. Helix path tracking of the quadrotor. 


\section{Conclusion}

In this research the full state dynamic equations of the quadrotor was derived. The quadrotor is an under-actuated system with 6-DOF and four propeller's speed as inputs. The relation between propeller's speed and trust force turned the angular speed to the force which was used as system input. Therefore, the main challenge was designing an impressive controller which was able to control all states. Three fuzzy logic controllers were designed to control all states. Finally, results were presented for both regulating the desired altitude and attitude and tracking the desired helix path. Also, helix path tracking of the quadrotor was presented.

\section{References}

[1] Bouabdallah, S. et al. (2004). "PID vs. LQ control techniques applied to an indoor micro quadrotor". Intelligent Robots and Systems, 2004.(IROS 2004).Proceedings.2004 IEEE/RSJ International Conference on, IEEE.

[2] Pounds, P., Mahony R., Corke P., (2006). Modelling and control of a quadrotor robot.In Proceedings of the Australasian conference on robotics and automation.

[3] Ahmad Mian, A., Wang Daobo, (2008), Modeling and Backstepping-based Nonlinear Control Strategy for a 6 DOF Quadrotor Helicopter, Chinese Journal of Aeronautics, Volume 21, Issue 3, Pages 261-268, ISSN 1000-9361

[4] Sangyam, T., Laohapiengsak P., Chongcharoen W., Nilkhamhang I., (2010), "Path tracking of UAV using self-tuning PID controller based on fuzzy logic". SICE Annual Conference 2010, Proceedings of, vol., no., pp.1265, 1269, 18-21 Aug.

[5] Santos, M.; López, V.; Morata, F., "Intelligent fuzzy controller of a quadrotor," Intelligent Systems and Knowledge Engineering (ISKE), 2010 International Conference on, vol., no., pp.141, 146, 15-16 Nov. 2010

[6] Luque-Vega, L., Bastillo-Toledo B., et al. (2011). "Robust block second order sliding mode control for a quadrotor." Journal of the Franklin Institute.

[7]Nagaty, A., Saeedi S., Thibault C., Seto M., Howard Li, (2013), "Control and Navigation Framework for Quad-rotor Helicopters.J. Intell Robot System.

[8] Rabhi, A.; Chadli, M.; Pegard, C., "Robust fuzzy control for stabilization of a quadrotor," Advanced Robotics (ICAR), 2011 15th International Conference on , vol., no., pp.471,475, 20-23 June 2011

[9] Bouabdallah, S.; Murrieri, P.; Siegwart, R., "Design and control of an indoor micro quadrotor," Robotics and Automation, 2004. Proceedings.ICRA '04. 2004 IEEE International Conference on, vol.5, no., pp.4393, 4398 Vol.5, 26 April-1 May 2004.

[10] A. Dzul P. Castillo and R. Lozano.Real-time stabilization and tracking of a four-rotor mini rotorcraft. IEEE Transaction on Control System Technology, 12(4):510 - 516, July 2004.

[11] R. Lozano P. Castillo and A. Dzul.Stabilization of a mini rotorcraft having four rotors.Proceedings of 2004 IEEE/RSJ International Conference on Intelligent Robots and Systems, pages 2693 - 2698, 2004.

[12] A. Palomino S. Salazar-Cruz and R. Lozano. Trajectory tracking for a four rotor mini-aircraft. Proceedings of the 44th IEEE Conference on Decision and control, and the European Control Conference 2005, pages $2505-2510$,

[13] R. Lozano P. Castillo and A. Dzul.Stabilization of a mini rotorcraft with four rotors. IEEE Control Systems Magazine, pages 45 - 55, 2005.

[14] A. Mokhtari and A. Benallegue. Dynamic feedback controller of Euler angles and wind parameters estimation for a quadrotor unmanned aerial vehicle. Proceedings of the 2004 IEEE International Conference on Robotics and Automation, pages 2359 - 2366, 2004. 
[15] A. Benallegue V. Mister and N. K. M'Sirdi. Exact linearization and noninteracting control of a 4 rotors helicopter via dynamic feedback. IEEE International Workshop on Robot and Human Interactive Communication, pages 586-593, 2001.

[16] M. Tarbouchi J. Dunfied and G. Labonte. Neural network based control of a four rotor helicopter. 2004 IEEE Intrnational Conference on Industrial Technology, pages 1543 - 1548, 2004. 2

[17] J. S. Jang S. L. Waslander, G. M. Hoffmann and C. J. Tomlin. Multi-agent quadrotor testbed control design: Integral sliding mode vs. reinforcement learning.

[18] T. Hamel N. Guenard and R. Mahony. A practical visual servo control for a unmanned aerial vehicle. 2007 IEEE International Conference on Robotics and Automation, pages 1342 - 1348, 2007.

[19] M. Valenti G. P. Tournier and J. P. How.Estimation and control of a quadrotor vehicle using monocular vision and moire patterns.AIAA Guidance, Navigation, and Control Conference and Exhibit, 2006. 2

[20] T. Hamel N. Metni and F. Derkx.Visual tracking control of aerial robotic systems with adaptive depth estimation.Proceedings of the 44th IEEE Conference on Decision and Control, and the European Control Conference 2005, pages 6078 - 6084, 2005. 2

[21] Zadeh, L.A., (1965), Fuzzy sets, Information and Control, Volume 8, Issue 3, June, Pages 338-353, ISSN 0019-9958.

[22] Kazemi. M, Alimi. A, "A fully fuzzy approach to data envelopment analysis", the journal of mathematics and computer science, Vol. 11, Issue 3, PP. 238-245, 2014.

[23] Almasi. O.N, Fereshtehpoor. V, Zargari. A,Banihashemi. E, "Design an Optimal T-S Fuzzy PI Controller for a Non-Inverting Buck-Boost Converter", the journal of mathematics and computer science, Vol. 11, Issue 1, PP. 42-52, 2014.

[24] Gheibi. A, Mohammadi. S. M. A, Farsangi. M. M, "Comparing performance of PID and fuzzy controllers in the present of noise for a Photovoltaic System", the journal of mathematics and computer science, Vol. 9, Issue 1, PP. 69-76, 2014.

[25] Bresciani, T. Modelling, "identification and control of a quadrotor helicopter", Department of Automatic Control, Lund University, (2008).

[26] Rodić A., Mester G., "The Modeling and Simulation of an Autonomous Quad-Rotor Microcopter in a Virtual Outdoor Scenario", ActaPolytechnicaHungarica 8 (4) 